\title{
La dinamica dell'alta atmosfera $(*)$
}

\author{
S. GIORGI
}

Ricevuto il 19 Febhraio 1963

Rrassusto. - Viene preso in esame il problema della determinazione dei venti nellalta atmosfera. usando la tecnica della nube di Sodio-Litio, e viene presa in considerazione una rassegna dei diversi metodi più comunemente usata nei vari paesi. Particolare attenzione è rivolta al metodo italiano che viene illustrato in alcmi dettagli allo scopo di fornirem a completa soluzione del problema.

Sumary. - The problem of the winds determination in the high atmospliere by the Sodium-and-Lithium cloud technique is considered and a review is presented of the data reduction methods most commonly used in various countries. Particular attention is devoted to the italian method which is illustrated in some details in order to provide a complete solution to the problem.

Lo studio delle caratteristiche, sia dinamiche che strutturali, dellabla atmosfera viene oggi prevalentemente compinto mediante indagine diretta: a questo seopo i mazi sonda appaiono i mezzi più adatti.

Fino a quote dell'ordine di $60-70 \mathrm{~km}$ il campo si puo considerare arequisito ai normali mezzi di indagine meteorologiea (palloni sonda, mazi meteorologici, exce.). A quote molto alte invere - per fissare le idee al di sopra dei $250 \mathrm{~km}$ - sono i satelliti gli strumenti teconice più irlonei allo seopo. Rimane quindi un vasto ampo di quote in aui i razzi sonda operano convenientemente per la ricerca e lo studio dei parametri dinamici e struttumali dell'atmosfera.

Venendo a trattare piu specificatamente delle earatteristiche dinamiche, si puo dire che la tecrica più conveniente do quella della nube di

$(*)$ Xotal presentatal al 20 Congresso Interuazionale 'Teenico scientifieo dello sipario. Roma, 19-23 (iiumo 1962. 
sodio e litio a causa di alcuni fondamentali vantaggi che essa presenta rispetto ad altri metodi:

a) possibilità di riavare dati in 111 ampio intervallo di quote;

b) possibilità di seguire otticamente la nube da terra per un tempo sufficientemente lungo;

a) semplicità del calrico pagante con conseguente aumento della "reliability".

Per il punto e) chiarirà l'assunto una breve descrizione del carico pagante. Fsso è costituito da un contenitore dove è immessa una sostanza capace di autocombustione - termite, ad es. - e sodio polverizzato con una quantità addizionale di litio (sotto forma, quest'ultimo, di masserelle diffuse nella massa maggiore, o in condizioni analoghe al sorlio, ma in quantità minore) mescolati assieme.

Lin comando a tempo innesca la combustione della temite, il sodio (e il litio) viene polverizzato e fuoriesce attraverso apposite aperture praticate nelle pareti del contenitore, mentre il razzo continua nel suo moto ascensionale. Generalmente la quantità della miscela è tale (e la rombustione è regolata in modo) che il sodio fuoriesce fra mal quota di $75-80 \mathrm{~km}$ e la quota massima raggiungibile dal razzo (e, a volte, anche per una parte della traiettoria discendent( ). Questo spiega, sia detto per inciso, anche il primo dei vantaggi sopra elencati.

Il sodio ed il litio formano una nube artificiale che è illuminata dai raggi del sole e che, a cousa di complessi fenomeni fisico-chimici, di cui in questa sede si tralaseia un tentation di interperetazione, può essere fotografata da terra sotto vari angoli e posizioni.

Tali riprese fotografiche, e questo risponde al punto b) sopra ricordato, possono essere effettuate per un periodo di tempo abbastanza lungo, da venti a trenta minuti, perehè la diffusione della nube artificiale, benchè lapida a causa della assai larefatta atmosfera circostante, avviene in assenza, o quasi, di reazioni chimiche, in quanto, appunto, il numero di molecole di aria per unità di volume alle quote interessate dagli esperimenti è estremamente ridotto. Questo spiega anche perchè lat tecnica della nube di sodio e litio non possal essere impiegata a basse quote, praticamente — secondo esperienze condotte qualche tempo fa — al di sotto dei 69-70 km; infatti, a causa della densità atmosferiea relativamente alta, si innescano delle complesse reazioni chimiche con quasi immediata sparizione della nube artificiale. 
A scopo di confronto si può accenmare brevemente agli altri metodi più usati per lo studio diretto delle caratteristiche dell'alta atmosfera:

1) Sfere carlente (falling sphere).

In questo caso il carico pagante del razzo vettore c̀ costituito da un contenitore in ('ui è immesso un tessuto leggerissimo, ma abbastanza resistente, che, espulso dal suo alloggiamento alla quota voluta, si gonfia assumendo la forma di ma sfera. Tale sfera, che in genere ha mn diametro sui $30 \mathrm{~m}$ e vieme seguita da terra a mezzo ladar, cade lentamente verso il suolo - a causa della sua estrema leggerezza - mentre la romponente orizzontale dei suoi spostamenti permette la determinazione dei venti in quota (che sono -.- come meglio si speciticherà nel seguito -essenzialmente orizzontali). Questo metodo però può essere usato, come appare evidente, soltanto a quote non molto elevate - praticamente non oltre i $100 \mathrm{~km}$ - perchè la densità atmosferica deve essere abbastanza alta da impedire ma troppo rapida caduta della sfera.

\section{2) (irrumete esplosion.}

Durante la corsa ascensionale del razzo vettore si fammo esplodere ad intervalli prestabiliti di quote delle granate ad alto esplosivo, costituenti il carico pagante. E necessario disporre a terra di un preciso sistema d'inseguimento elettronico (Dovap, ad es.) e di un completo impianto acustico (microfoni di alta precisione, ad es.). I venti vengono valutati mediante una teconica abbastanza complessa e possono essere determinati solo come valore medio fra due quote sucessive di esplosione delle granate. Si tratta quindi di un metodo non molto preciso, anche se abbastanza mapido, che non può dare ragione di molti importanti fenomeni dinamicei dell'alta atmosferica ("shear"), ar esempio).

3) Nubi artificiali di metalli alcalini. quali cesion e potassio.

La tecnica d analoga a quella delle esperienze sodio-litio, salvo che la nube ionizzata che si forma non emette nel visibile e deve essere seguita da ter"a mediante "tracking " ladarice; essal richiede cioè un'attrezzatura assai complessa e costosa. Peraltro, questo metodo, permettendo i lanci in ogni ora del giomo, presenta un vantaggio rispetto alla tecnica del sodio-litio che, come noto, puo essere impiegata solo in esperienze com- 
pinte poro prima dell'alba o poco dopo il tramonto. Infatti, mentre la nube deve essere "illuminata " dal sole, le stazioni di ripresa a terra nom devono essere investite dalla luee solare né diretta, né diffusa dall'ambiente circostante, per evitare di "annegare" sulla lastea l'immagine dolla nube.

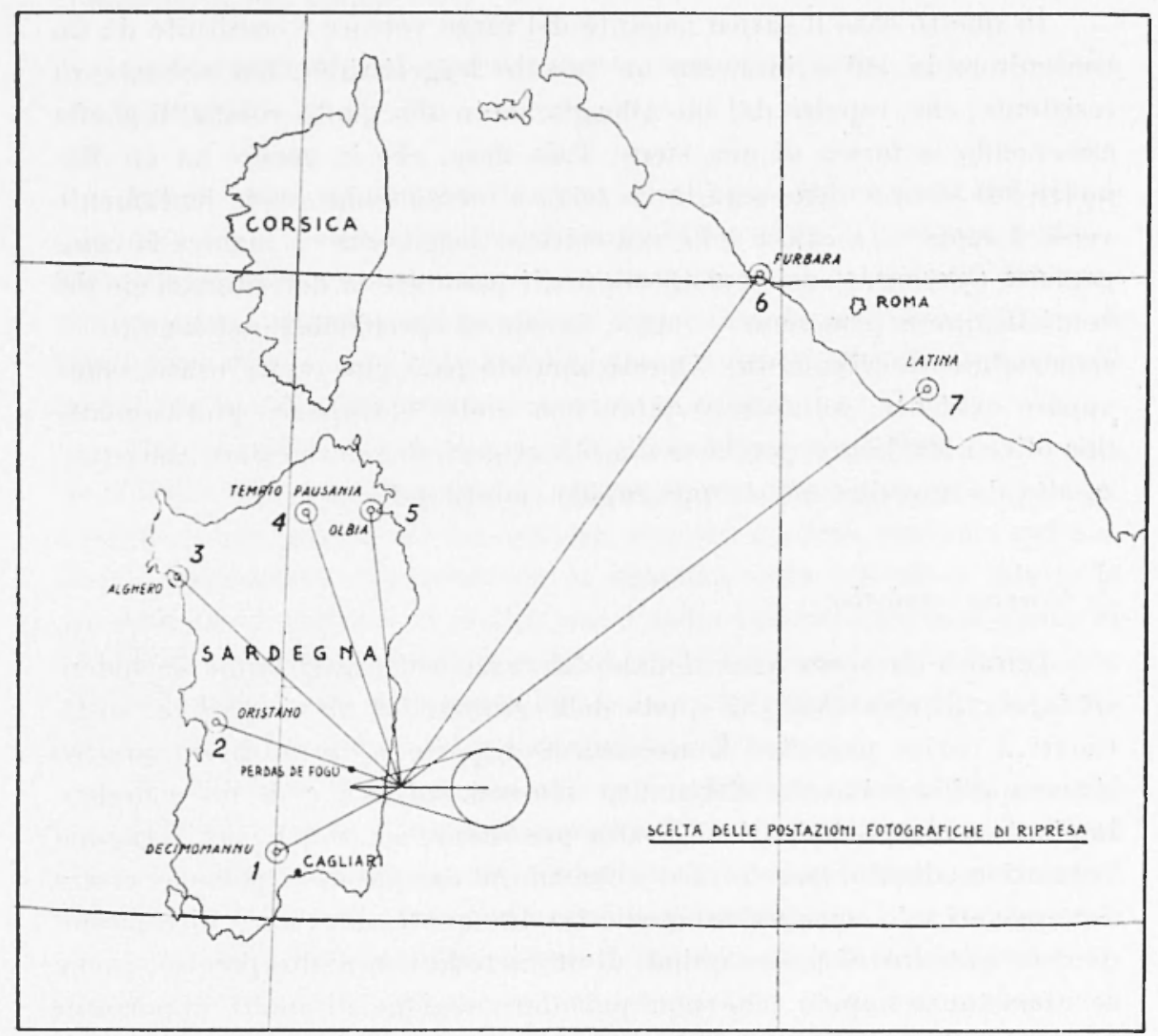

Fig. 1

Sel programma italiano di ricerehe nellalta atmosfera srolto dalla Commissione per le Ricerehe Spaziali presieduta dal Prof. Broglio è stata impiegata la combinazione razzi sonda-mubi di sodio e litio e la distribuzione delle postazioni fotografiche a terra is stata finora quella riportata in Fig. 1. Tali postazioni, disposte intorno al Poligono del Salto di Quira da cui sono stati eflettuati i lanci, sono state dotate di quattro marechine fotografiche di ripresa riascuna di diverse calratteristiche. 
Le macehine scattano i fotogrammi ad intervalli prestabiliti di tempo con tempi di esposizione parimenti lissati ed identici per tutte le macchine di tutte le stazioni. Sempere pere i lanei italiani si riportano, a titolo di esempio, due sequenze di tali fotoglammi (Figg. 2, 3, 4, 5, 6, 7 ). Nelle due ultime figure sono articolarmente pevidenti gli "shear m. Il fenomeno degli "shear" cioè di correnti, o renti, dell'alta atmosfera che in un intervallo relativamente ristretto di quote ambiano bruscamente dilezione, è uno dei più importanti della dinamio"a dell'alta atmosfera. La Fig. 8, che si riferisee ad un lancio differente di quello relativo alle Figg. 6 a 7 , ne dà un altro esempio. Nella Fig. 8 è possibile indiviluare un altro importante fenomeno dinamico: la turbolenzal, di cui la Fig. 9, particolare della precedente, dà un quadro più chiaro.

Si notano in particolare i filamenti di nube di cui appare l'insorgenza da un unico centro sul braceio principale della nube stessa. Il fenomeno della turbolenza negli esperimenti finoma condotti, in Italia ed in altri Paesi, si è verificato a quote piuttosto basse: secondo alcuni ricereatori il limite superiore dovrebbe essere fissato a $102 \mathrm{~km}$. Questo fatto è confermato dai risultati sperimentali che hanno indicato la presenza di turbolenza solo fra 80 e. $100 \mathrm{~km}$. Da $80 \mathrm{~km}$ in su sono anche presenti gli "shear", il cui campo si estende, almeno nella quasi totalità dei casi, fino a $110-115 \mathrm{~km}$ e non oltre.

I fotogrammi sono gli elementi di base per la lestituzione dei dati. In questa fase degli esperimenti il problema principale è quello della individuazione dei punti omologhi, cioè dei punti su due o. più fotogrammi diversi, ma presi allo stesso istante in due o più differenti postazioni, che corrispondono allo steso punto (particelle) della nube velua. La restituzione dei dati, dioè la ricostruzione della posizione spaziale vela della nube ad ogni istante, è possibile quindi solo disponendo delle serie complete di fotogrammi resative ad almeno due postazioni di ripresesa.

Ritomando ora al problema principale si può dire che sono stati coscogitati vari metodi per la determinazione dei punti omologhi. Intanto se ne possono citare almeno due che "aggirano "la difficoltà:

a) licostruzione fotogrammetrica (stereofotogrammetria);

b) uso di stazioni fotografiche di ripresa molto lontane dal punto di lancio.

Ma essi sono troppo poco precisi per gli scopi dell'esplorazione dellalta atmosfera. 
I metodi effettivamente impiegati sono vari; se ne possono ricordare tre:
1) metorlo amerieano: analogico
2) metodo inglese : numerice
3) metodo italiano : analitico.

Il metodo americano è basato sulla ricostruzione in seala in un rasto ambiente a terra del fenomeno reale. Al posto delle stazioni fotografiche vi sono dei proiettori il cui asse ottico d̀ orientato identicamente a quello delle macchine fotografiche. Nel modello le negative dei fotogrammi sono proiettate su un grande schermo emisferico (Fig. 10). Una disposizione opportuna delle lenti dei proiettori assicura che tutti gli angoli del modello siano conservati rispetto al fenomeno reale.

In queste condizioni un sottile filo di nylon (o simile materiale) tirato fra un proiettore del modello ed un punto della corrispondente immagine proiettata dalla nube rappresenta ma "linea di vista " fia la macehina fotografica corrispondente al proiettore a lo stesso punto (particella) della nube vera. Un secondo filo timato da un secondo proiettore, sempre nel modello, allo stesso punto della corrispondente immagine della nube incrocia il primo filo in un punto che corrisponde nel modello alla posizione reale della particella della nube vera. Per migliorare la precisione si considerano anche gli altri proiettori in modo da ottenere un incrocio fra tanti fili quante sono le stazioni di ripresa. Essendo fissata c nota la scala del modello, è possibile allora ricostruire completamente la posizione della nube ad ogni istante.

Il metorlo richiesle dunque una complessa apparecehiatura a terra, il rhe costituisce certamente uno svantaggio; inoltre l'individuazione dei punti omologhi avviene, soma sopra accennato, per vie prevalentemente " visive" e questo è causa di una certa imprecisione soplattutto nella zona di nube con pochi elementi caratteristici, cioè negli intervalli di quote in cui il vento è quasi costante in intensità e direzione: ('io si verifica in particolare alle alte quote - per fissare le idee, al di sopra dei $150 \mathrm{~km}$.

Nel metodo inglese si considerano per ogni istante le coppie di fotogrammi contemporanei ottenuti in due stazioni diverse. Si fa inoltre riferimento, per semplieitid all'asse centrale dell'immagine della nube. Su tale asse si individuano per ciasceun fotogramma un certo numero di punti (cinquanta, ad es.). Si considera poi un punto su di un fotogramma e si determina analiticamente la retta congiungente tale punto col centro ottico della marechina che ha ripreso il corrispondete fotogramma. In modo analogo si costruiscono le rette passanti per il centro ottiro 
dell'altra macehina e per tutti i punti segnati sullalt ro fotogramma: fra tali rette si cerea poi quella che intersecal la retta del primo foto-

\section{RESTITUZIONE DEI DATI \\ METODO AMERICAMO}

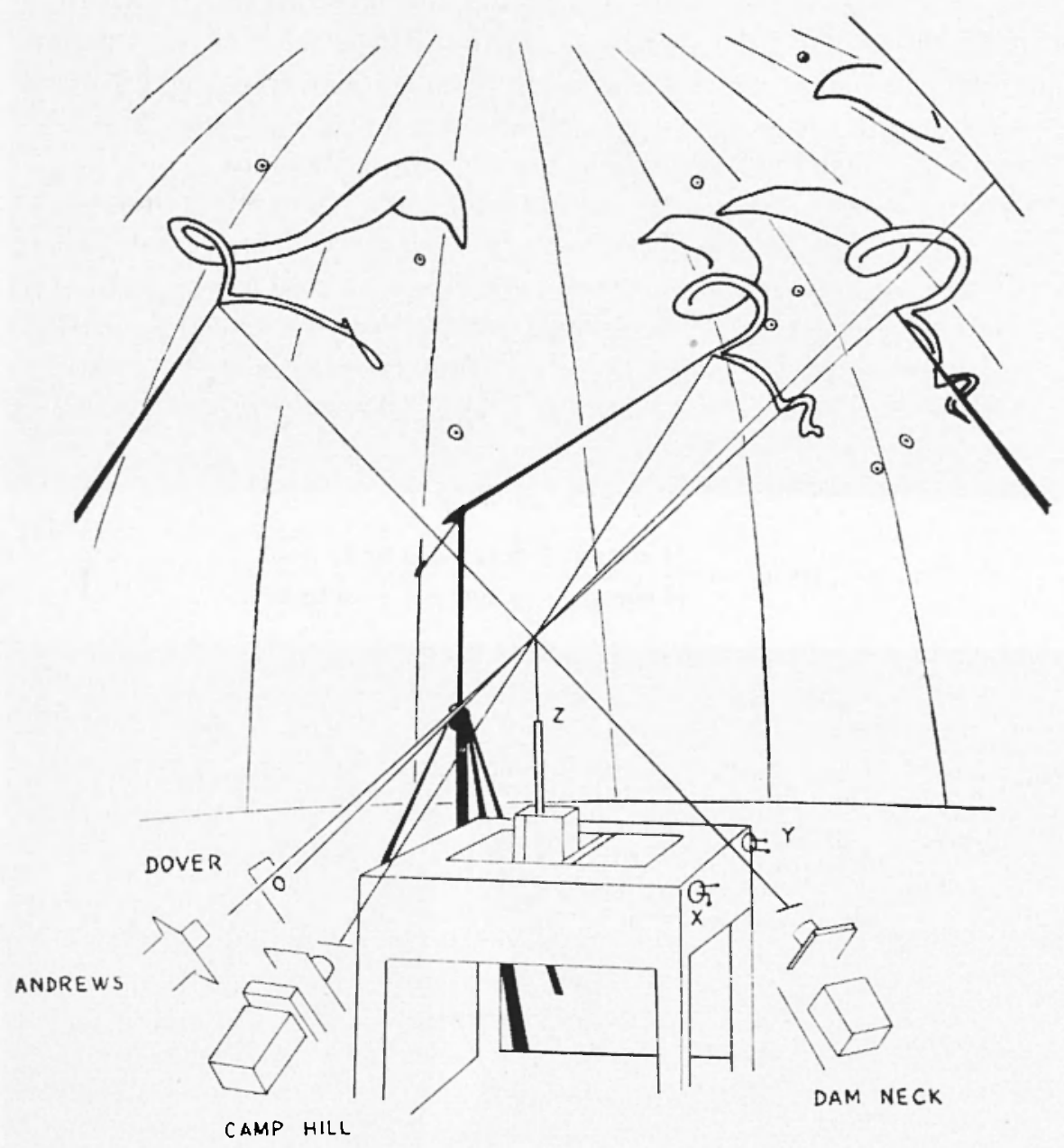

Fig. 10

gramma o celue passa alla minima distanza da essa. Si determina cosi nel secomclo caso mediante ma opportuna approssimazione - la posizione spaziale vera di un punto della nube. 
Ripetendo lo stesso procedimento per tutti i punti del primo fotogramma si viene a ricostruire la nube, o meglio il suo asse rentrale (*), al ogni istante.

ì evidente nel metodo ma reeta mancanza di precisione, ma, soprattutto una notevolissima laboriosita che allunga di molto i calcoli necessatri, anche ricorrendo all'uso di calcolatori digitali.

Il metodo italiano è stato sviluppato dal Prof. Broglio in occasione delle serie di lanci dal Poligono del Salto di Quirra. Si ronsiderino due fotogrammi presi allo stesso istante in due stazioni diverse. Ogni punto dell'immagine della nube - per semplicità si può fare riferimento all'asse centrale - i individuato da due coordinate di lastra $x, y$.

Si consideri poi il piano verticale a passante per le due stazioni di rui sopra e per ognuna di esse si tissi un sistema di coordinate polari sferiche con origine nel centro ottico di ciascuma stazione e in cui l'elevazione $\varphi$ è contata a partire dall'orizzontale locale e l'azimut 0 a partire dal piano $a$. Le coordinate polari $q$ e $\theta$ sono legate alle roordinate di lastra $x, y$ (Fig, 11 ) dalle semplici relazioni trigonometriche:

$$
\begin{aligned}
& \operatorname{sen} \varphi=\frac{f \operatorname{sen} \varphi_{0}+y \cos \varphi_{0}}{\sqrt{f_{0}+x^{2}+y^{2}}} \\
& \operatorname{tg} 0=\frac{\left(f \cos \varphi_{0}-y \operatorname{sen} \varphi_{0}\right) \operatorname{tg} \theta_{0}+x}{\left(f \cos \varphi_{0}-y \operatorname{sen} \varphi_{0}\right)-x \operatorname{tg} \theta_{0}}
\end{aligned}
$$

COORDINATE DI LASTRA E COORDINATE POLARI

Fig. 11

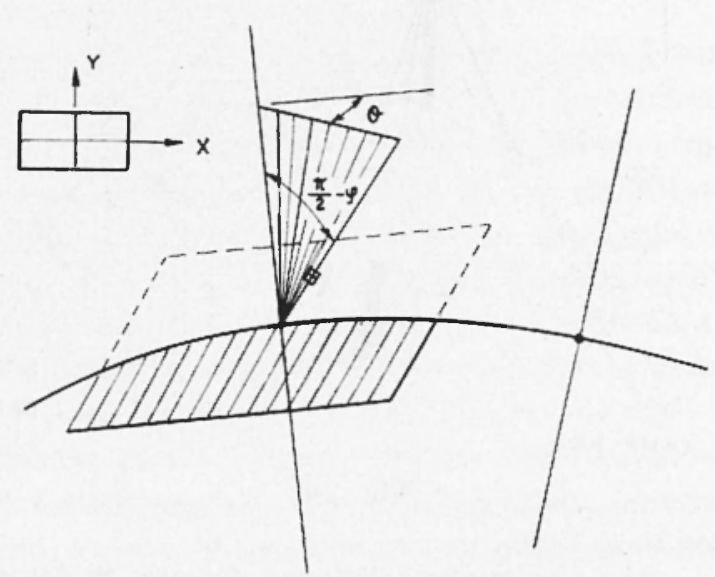

(*) Dal punto di vista delle caratteristiche dinamiche, il limitarsi alla considerazione dell'asse centrale non comporta in pratica alem errore sensibile. 


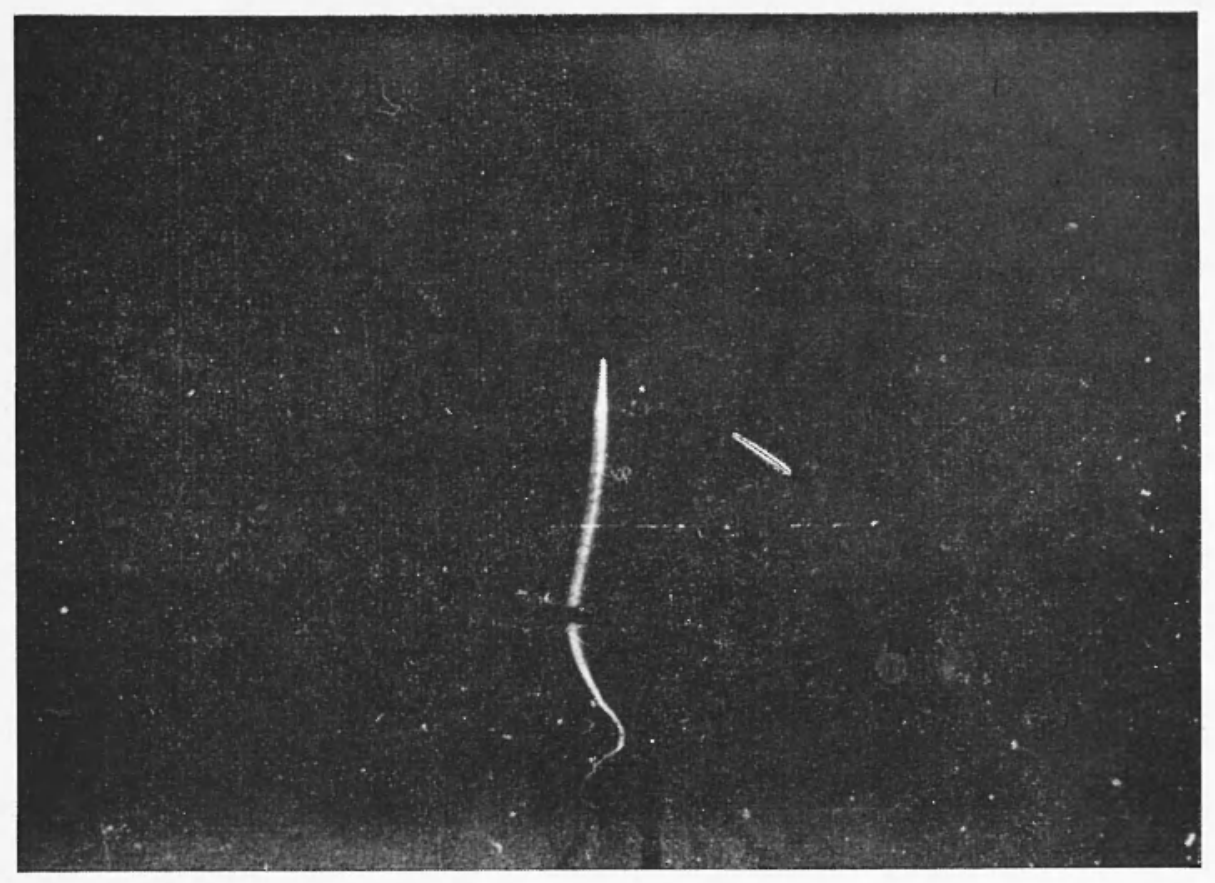

Fig. 2

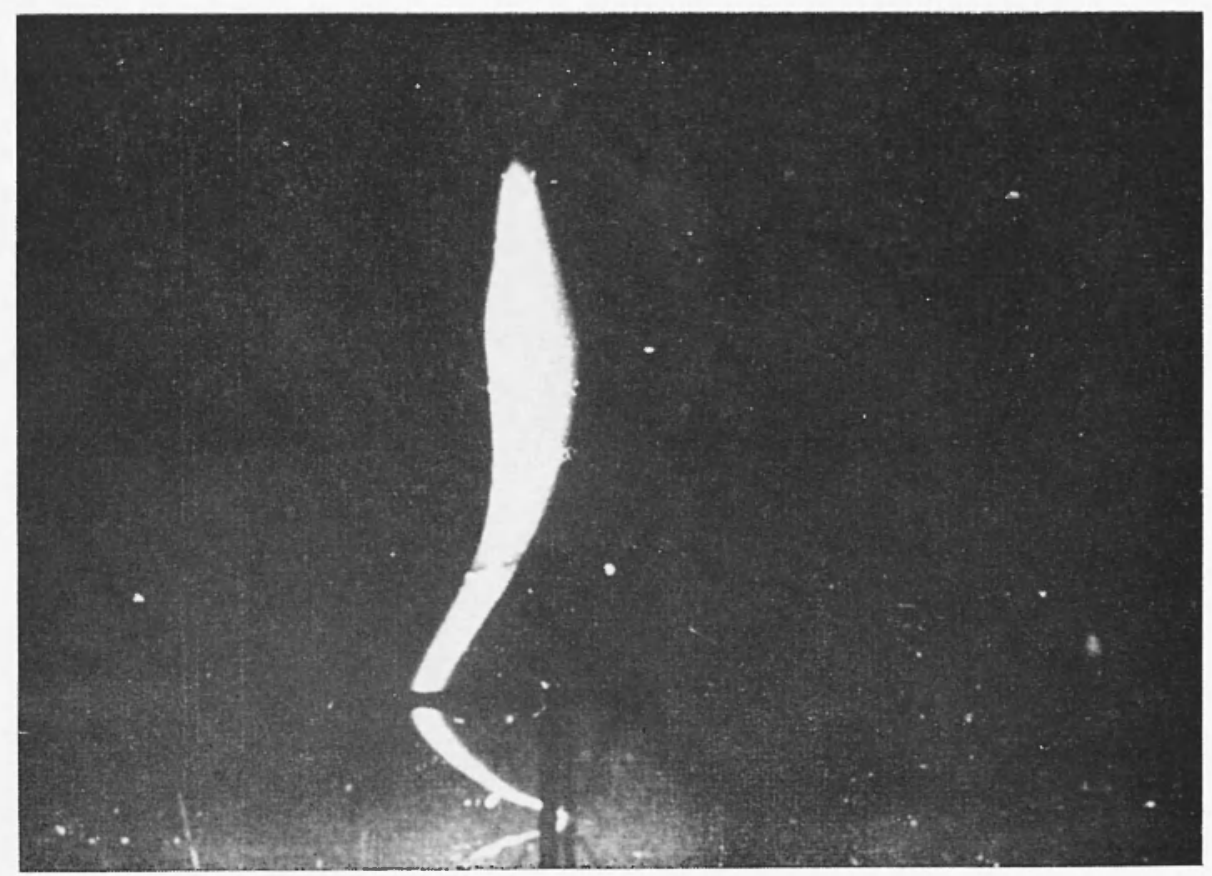

Fig. 3 


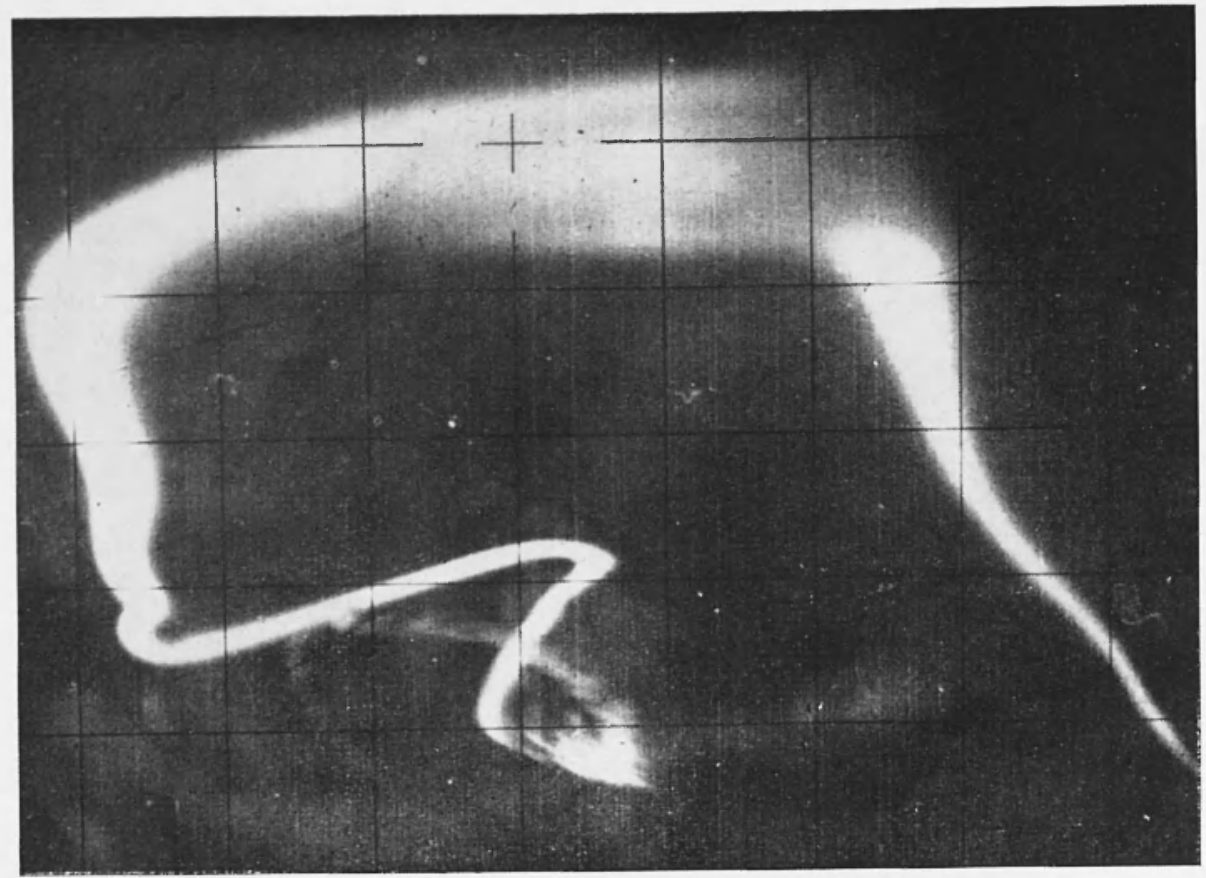

Fig. 8]

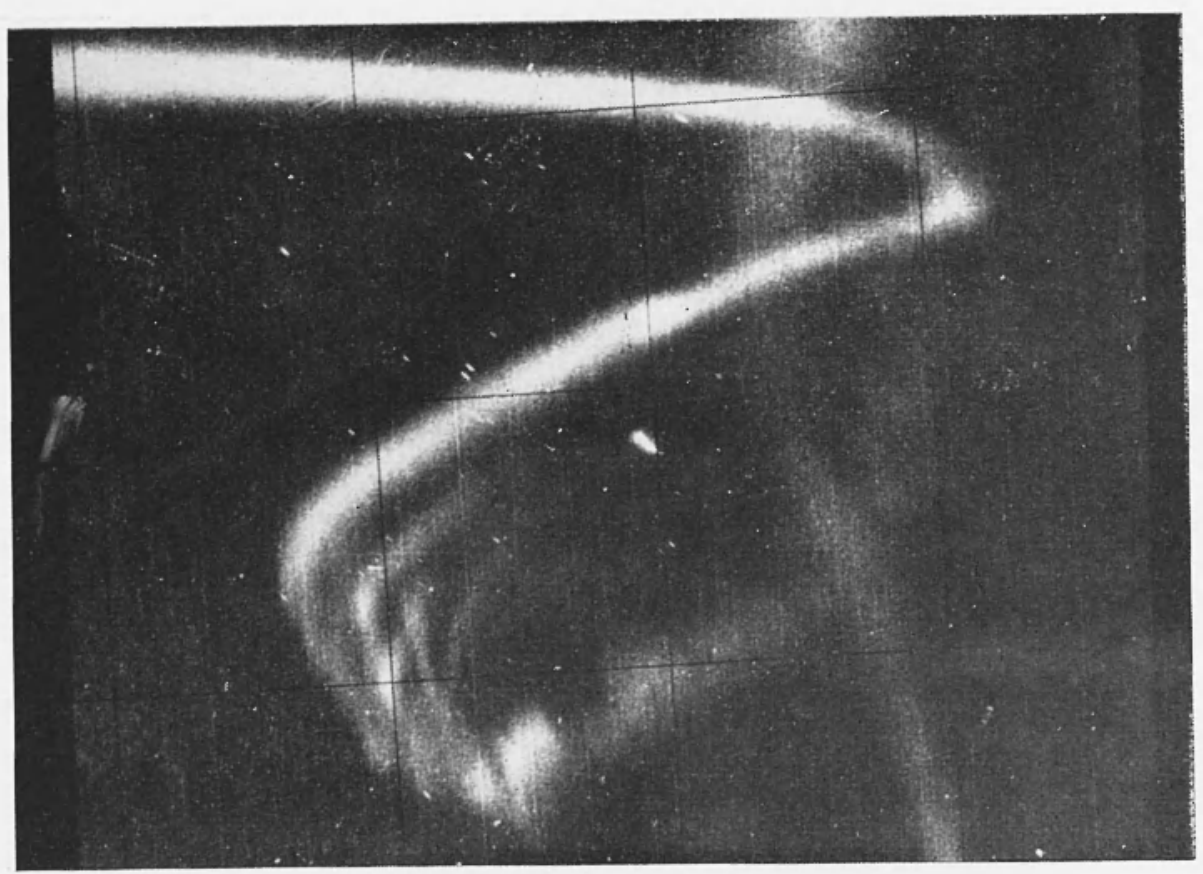

Fig. 9 
dove $f$ ì la distanza forale e $\varphi_{0}, O_{0}$ elevazione ed azimuth dell'asse ottico (quantità, questè, note).

Ciascuu punto della nube vela, essendo allineato con il suo punto immagine e col centro oftico, is definito quindi mediante le stesse $q$ e 0 dello stesso punto immagine, rimanendo in questa fase del procedimento ancora incognita la distanza $Z$.

D'alta parte un punto della nube puo anche essere determinato mediante gli angoli $\xi$ e $\zeta$ (Fig. 12), legati a $q$ (c) 0 dalle relazioni:

$$
\begin{aligned}
& \operatorname{tg} \xi=\frac{\operatorname{sen} i}{\operatorname{tg} \theta}-\frac{\operatorname{tg} \varphi}{\operatorname{sen} \theta} \cos i \\
& \cos \xi=\cos \varphi \cos \theta \cos i+\operatorname{sen} \varphi \operatorname{sen} i
\end{aligned}
$$

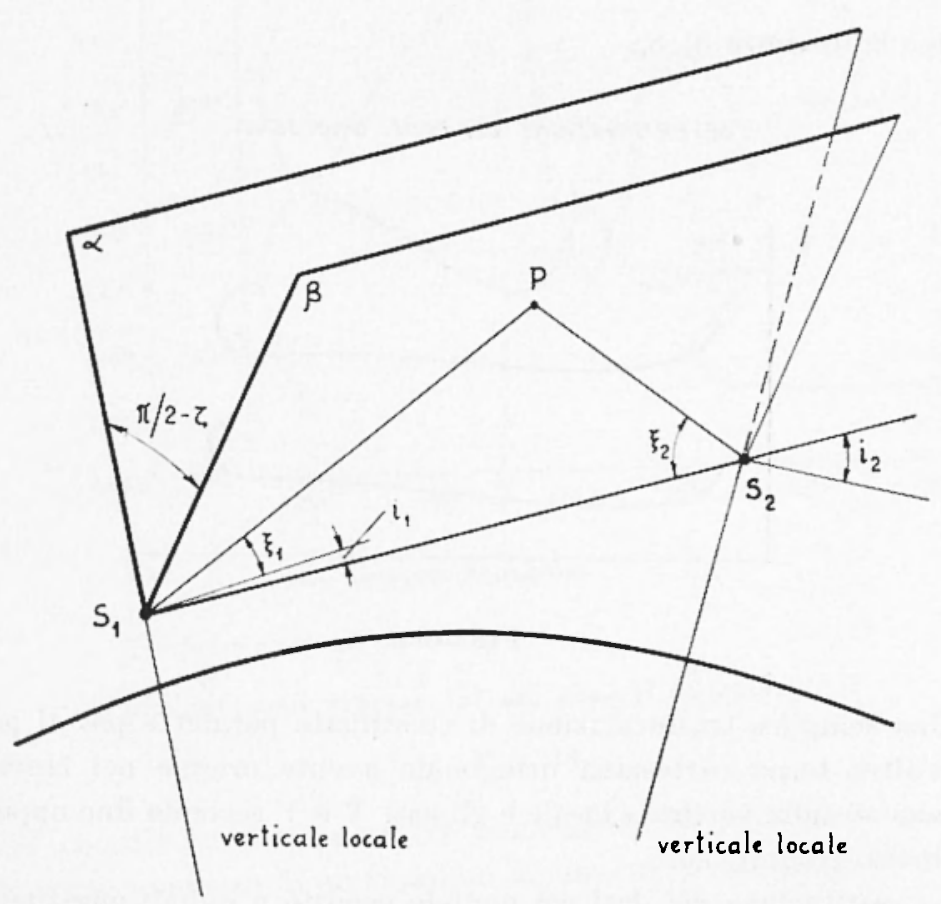

Fig. 12

dove $i$ i loinclinazione della orizzontale locale pispetto alla congiungente $s_{1} S_{2}$.

Effettuando le operazioni per diascun punto dell'asse centrale della nube sui fotogramani si ottengono le due curve $\xi_{1}(\zeta)$ e $\xi_{2}(\xi)$ (Fig. 13). Ne risulta immediata la deteminazione dei punti omologhi: infatti ogni 
punto $P$ della nube $\dot{e}$ definito dallo stesso ${ }^{-}$per ciascuna delle due stazioni. Facendo riferimento ad un sistema cartesiano ortgogonale di riferimento con origine, ad es., nel punto $S_{1}$, reon l'asse $Z$ orientato secondo la verticale locale, l'asse $Y$ sull'intersezione del piano orizzontale locale col piano a e l'asse $X$ in ronseguenzal, si ottiene allom:

$$
\begin{aligned}
& X\left(I^{\prime}\right)=b \frac{\operatorname{sen} \xi_{0}}{\operatorname{sen}\left(\xi_{2}-\xi_{1}\right)} \operatorname{sen} \xi_{1} \cos \zeta \\
& Y\left(I^{\prime}\right)=b \frac{\operatorname{sen} \xi_{2}}{\operatorname{sen}\left(\xi_{2}-\xi_{1}\right)}\left[\cos \xi_{1} \cos i_{1}-\operatorname{sen} \xi_{1} \operatorname{sen} i \operatorname{sen} i_{1}\right] \\
& Y\left(I^{\prime}\right)=b \frac{\operatorname{sen} \xi_{2}}{\operatorname{sen}\left(\xi_{2}-\xi_{1}\right)}\left[\operatorname{sen} \xi_{1} \operatorname{sen} \zeta \cos i_{1}+\cos \xi_{1} \operatorname{sen} i_{1}\right]
\end{aligned}
$$

dove $b$ is la distanza sis.

DETERMINAZIONE DEI PUNTI OMOLOGHI

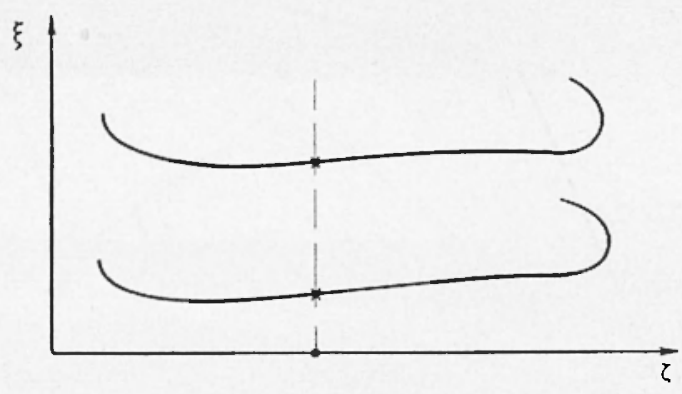

Fig. 13

Ena semplice trasformazione di coordinate permette poi di passare and un'altrat terna cartesiana ortogonale avente origine nel lanciatore, con l'asse $Z$ sulla verticale locale a gli assi $X$ e $Y$ secondo due opportune roordinate geografiche.

La restituzione dei dati col metodo esposto a quindi eftettuata con una elevata precisione non raggiungibile negli altri metodi. Anche lat alaborazione numerion ì sufficientemente rapicla: per i lanci italiani essa ì stata condotta presso l'Istituto di Costruzione Aeronatutiche dell'Universita di Roma mediante un calcolatore digitale Bendix ( $\mathrm{i}-1.5 \mathrm{D}$ ). Si noti infine rhe nel metodo italiano non è richiesto alcun complesso impianto sperimentale a terra a ciò costituisee un evidente rantaggio rispetto, and escompio, al metodo ammericano. 
La Fig. 14 mostra come in mo caso reale si presentino le curve $\xi_{1}\left(\begin{array}{c}6 \\ \text { ) }\end{array}\right.$ e $\xi_{2}(\zeta)$. I ralori estremi di $\zeta$ (o cos $\zeta$ ) somo gli stessi per le due rurve, come è logieo, dato che l'angolo dietro avente come vertice la congiumgente $S_{1} S_{2}$ e contenente tutta la nube deve essere lo stesso perr le due stazioni. Per la stessa ragione le tamgenti alle curve palallele alloasse

\section{DIAGRAMMA PER LA DETERMINAZIONE DELLE COPPIE}

DEI PUNTI CORRISPONDENTI

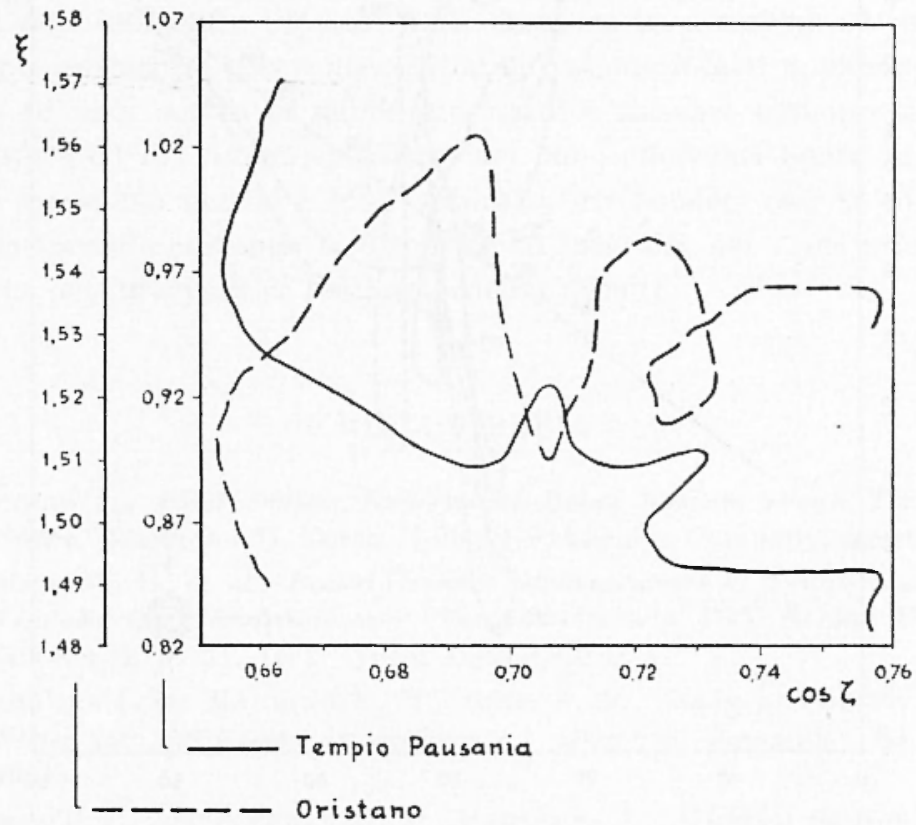

(Fotogrammi ripresi $151 \mathrm{sec}$. dopo il lancio)

Fig. 14

delle roomblinate devono essere roincidenti. Si noti infine la temdenza alla colrispondenza di un massimo di una curva ron un minimo dell'alt l"a a vicerersa.

Una rolta ceffettuata la restituzione dei dati, il procedimento per il calcolo dei renti è molto semplice ed ì concordemente arlot tato dai ricer(atori dei vari Paesi. Si fa la proiezione quotata al istanti suceessivi della nube sul jiano orizzontale passante per il lanciatore e si riporta sullo stesso diagramma la traiettoria del razzo restore ricarata in prereslonzil. 
La Fig. 15 mostra il procedimento in un caso reale (sul diagramma sono ripoltate, per mgioni di chianezza, solo due curve). Ola, i venti alle alte quote sono fondamentalmente orizzontali e costanti in direzione ad intensita, almeno per un tempo sufficientemente breve quale quello interessito diall'esperimento.

\section{PROIEZIOME ORIZZOMTALE DELLA MUBE}

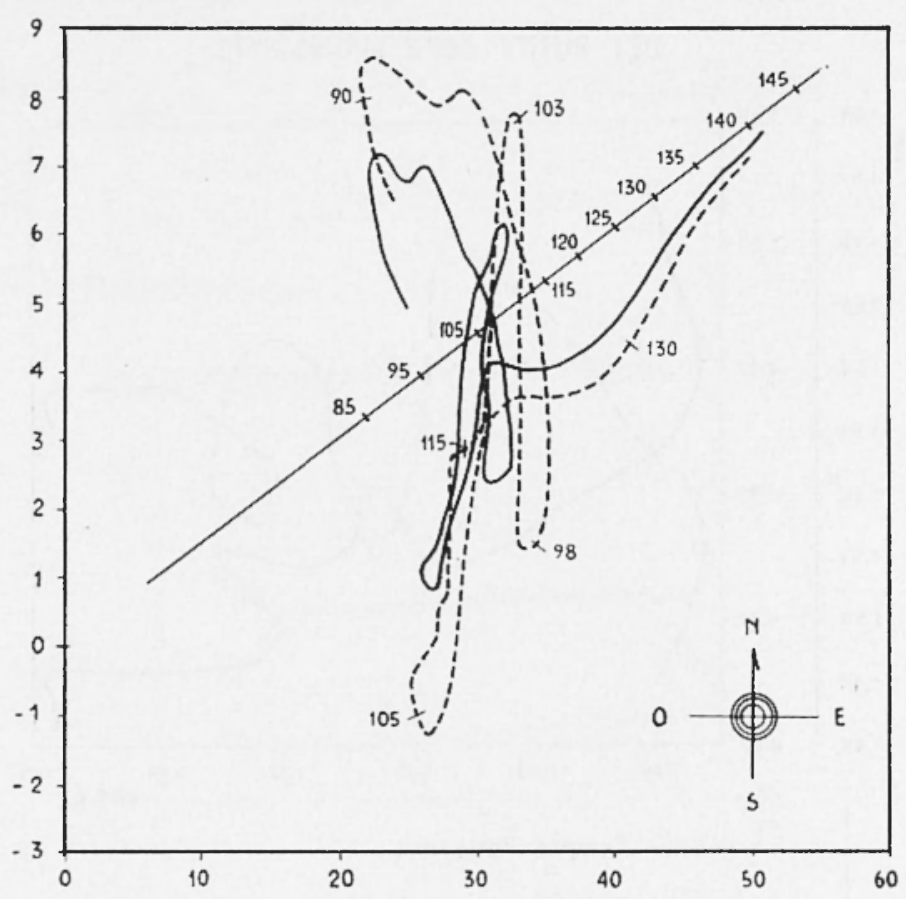

fotogramma ottenulo 151 sec. dopo il lancio

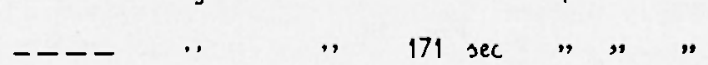

Fig. 15

In queste condizioni i punti della traiettoria del razzo e delle proiezioni della nube ad istanti suecessivi aventi la stessa quota risulano allineati su una retta. So si indica allora con $x_{1}, y_{1}, z$ un punto su mol curva (relativa al tempos $t_{1}$ ) e con $x_{2}, y, z$ un punto su un'altra curva (relativa al tempo $t_{2}$ ), lo spostamento $D$ i:

$$
D(z)=\sqrt{\left(x_{2}-x_{1}\right)^{2}+\left(y_{2}-y_{1}\right)^{2}}
$$


e quindi la relocità del vento

$$
V(z)=\frac{L(z)}{t_{2}-t_{1}}
$$

mentre la slat diregione:

$$
\operatorname{tg} O(z)=\frac{y_{2}-y_{1}}{x_{0}-x_{1}}
$$

avendo orientato l'asse $x$ verso est a lausse y verso nord.

Si noti infine che l’ipotesi della costanza in direzione ed intensiti dei venti orizont.uli non i necessaria: si possono infatti applieare le [4]. [5], [b] ad ogni ropplia di curve sucesessive e ricavare così le variazioni di $V(z)$ e $O(z)$ nel tempo; peralter, nei lanci effettuati finora in Italia, questo fenomeno nom si i mai rerificato, ottenendosi cosi la conferma della sostanziale costanza in clirezione ed intensita dei renti orizzontali in quota pere intervalli di tempo di alcuni minnti.

\section{BIBLIOCRAFLA}

(') Brotino I., First Italian Experiment Using Sodium Olond Technique. "Space Researeh" II North Holland Publishing Company, Amsterdam.

${ }^{2}$ ) Stroud W. G. et al., Rocket-Grenade Measurements of Temperatures and Winds in the Mesosphere over Churchil. "Canada. IOY Rocket Report", Number 6, p. 31. IGY World Data Center A.

(3) Bedisier .I. F., Maxisa E. R., Gosir S. N.. Study of Sodium Frapor Ejected into the Cpper Ltmosphere. "J. Geophys. Research ", 63, 19-29, (1958).

(") Marmo F. F., Aschemiskaxi Т. M., Pressman J., Lrtificial electron clouds. Cinque pubblicazioni in "Planetary and space Science ", Pergamon Press, Londrar da Agosto 1959 ad Aprile 1960.

(5) Buanox' J., Nuages Irtificiels de Sodium. "space Research I", North Holland P'ublishing Company. Amsterdam.

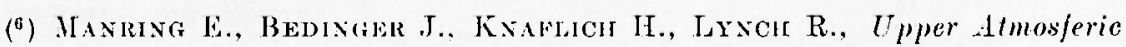
Wind Profiles Determined from three Rocket Experiments. GCA Technical Raport 61-1 - N. Geophysies Gorporation of America.

(i) Bromiso L., Review of Italian Meteorological Activities and Resulls. In corso di pubblicazione in "Space Research" III. North Holland Publishing Company - Amsterdam.

(s) BLAMoxt J., DE Jatien D.. Upper .tmospheric Turbulence Determined by ITeans of Rockets. "J. (Geophyss. Research ", 67, (9 July 1962). 